\title{
OTIONOMICS
}

Revista de economía, empresa y sociedad

Dossier «Prevención de riesgos laborales: tendencias en tiempo de crisis»

UN FACTOR CLAVE

\section{Integración del análisis coste-beneficio en la gestión de la prevención de riesgos laborales}

\section{Garles Salas Ollé}

Responsable de seguridad y salud laboral de Tusgsal. Profesor asociado del Departamento de Organización de Empresas de la Universidad Politécnica de Cataluña

RESUMEN La argumentación rentabilista de la gestión de la prevención de riesgos laborales supone una búsqueda del consenso y el pragmatismo entre los empresarios y los gestores de la prevención. La falta de experiencias en el uso de indicadores socioeconómicos preventivos, ya que esto por diferentes motivos no ha impregnado en los técnicos en prevención de riesgos, ocasiona temor y desconocimiento para decidirse a implementar este ámbito de la gestión y, por lo tanto, resulta complicado buscar paralelismos acerca de los datos registrados. Ello deja la gestión preventiva en un segundo plano frente al resto de las áreas de gestión en la mayoría de las organizaciones. El cálculo de costes de la accidentalidad en las empresas es un buen campo para comenzar a implementar la necesaria integración del análisis coste-beneficio de la seguridad y salud laboral en la gestión habitual de estas.

PALABRAS CLAVE gestión preventiva; análisis coste-beneficio; mejora continua

\section{Integration of the cost-benefit analysis in managing the prevention of occupational risks}

\begin{abstract}
The profitability approach in the management of the prevention of occupational risks entails a search for consensus and pragmatism among prevention entrepreneurs and managers. Since the lack of experiences in the use of preventive socioeconomic indicators has not impregnated risk prevention technicians for different reasons, it causes fear and ignorance when it comes to deciding whether to implement this field of management. Therefore, it is difficult to find parallelisms regarding the recorded data. This places preventive management in the background compared to other areas of management in most organizations. The calculation of costs derived from accidents in companies is a good field to start implementing the necessary integration of the cost-benefit analysis of occupational safety and health in the usual management of them.
\end{abstract}

KEYWORDS preventive management; cost-benefit analysis; continuous improvement 


\section{Introducción}

El análisis beneficio-riesgo de la gestión en general es algo intrínseco a cualquier sistema de gestión. El sistema de cálculo de costes de accidentalidad, como primer paso que dar en esta faceta, permite llegar a poder calcular de forma sencilla y razonada los gastos ocasionados por cada accidente de trabajo, lo cual permite considerar qué ahorramos al obtener un descenso de accidentalidad y, a su vez, valorar la rentabilidad económica de la gestión preventiva de forma directa. A partir de aquí, ahondar no resulta complicado, de manera que profundizando en esta dirección los costes generales de absentismo se pueden obtener representando también un indicador de gestión muy importante que hemos de tener en cuenta y que, además, debe orientar en la toma de decisiones acerca de las estrategias o medidas que tomar para su control o reducción.

Sin embargo, la gestión de la prevención de riesgos laborales, lejos de haberse situado en la primera línea de los ámbitos de gestión de las empresas, queda relegada, en la mayoría de los casos, a una segunda fila en este sentido.

\section{Análisis coste-beneficio}

El análisis coste-beneficio (ACB) de la gestión en las empresas es la clave para determinar el protagonismo o no que se le otorgue a un ámbito de gestión. Así, en la seguridad y salud laboral este ACB se presupone poco atractivo, por lo que no se ha procedido a realizar un análisis específico en este sentido.

La rentabilidad de las ratios relacionadas con la salud y la productividad o la competitividad o, más específicamente las relacionadas con la salud laboral, no es algo nuevo, sino que ya desde un tiempo a esta parte los economistas se preocupan y ocupan de conocer con el mayor detalle este tipo de relaciones.

De este modo, las decisiones que impliquen según qué tipo de inversiones pueden ajustarse a la medida de los condicionantes de cada momento y cada lugar. Esto es trascendental para el éxito de cualquier inversión económica.

Se han establecido en numerosos estudios macroeconómicos relaciones de positividad entre el nivel nutricional de un país y su PIB per cápita (Arcand, 2001) o un aumento en la esperanza de vida y el crecimiento económico de una nación (Bloom y otros, 2001).

Asimismo, el World Economic Forum en sus informes habituales (por ejemplo el de 2005-2006 citado en la bibliografía) incluye aspectos tales como el impacto del SIDA o el de la malaria o la tuberculosis en los negocios según los países; dando en dichos informes una mayor importancia al papel de la salud en la competitividad y admitiendo, además, que falta ampliar la visión sobre el sector salud.

Pese a las dificultades que pueda entrañar demostrar que hacer prevención es rentable y aumenta la competitividad de las empresas, existen estudios de carácter más específico, más que suficientes, llevados a cabo por instituciones de reconocido prestigio y solvencia como para poder lograr destacar tal evidencia.

Así, la Agencia Europea para la Seguridad y Salud en el Trabajo puso en marcha una campaña en el año 2010 para promocionar la salud en las empresas, a través de la cual se demuestra que, además de los beneficios de salud para los trabajadores, cada euro invertido en promoción de salud en el trabajo arroja un rendimiento de inversión de entre 2,5 y 4,8 euros, gracias a la reducción de los costes del absentismo fundamentalmente. Como ejemplo de esta campaña se cita un caso práctico trabajado en Dundee City Council (Reino Unido), donde se decidió llevar a cabo un plan para mejorar la salud psicológica y física de su personal en su Departamento de Trabajo Social. Esto lo planificaron con varias iniciativas centradas en acciones de carácter organizativo (políticas favorables a la familia) y medidas de acción individuales (como la facilitación de un servicio de asesoramiento confidencial a los empleados o la afiliación a un club de adelgazamiento subvencionada, etc.).

El plan consiguió una mejora de la salud de los empleados, y esta mejora se estima que produjo un ahorro de 165.000 euros en bajas laborales por año, fundamentalmente gracias a la reducción del estrés, la ansiedad y los problemas musculoesqueléticos. 
El propio director de la EU-OSHA manifiesta con relación a esta campaña que «Apoyar un estilo de vida sano beneficia a todos. Beneficia a los propios empleados y a sus empresarios, pero además al conjunto de la sociedad, reduciendo la carga sobre los sistemas de atención sanitaria. Y con el envejecimiento de la población laboral, va a seguir aumentando la importancia de los planes de PSLT, tenemos que ayudar a la gente a permanecer más tiempo sana, y para ello empresarios y trabajadores deben colaborar estrechamente, para crear una cultura de trabajo saludable». Ello quiere incidir sobre el envejecimiento de la pirámide demográfica, que está provocando ya que los trabajadores dejen de trabajar más tarde y, por lo tanto, ante la previsión de una masa no desdeñable de trabajadores de mayor edad, se deban plantear políticas y actuaciones de promoción de la salud y prevención para así obtener una relación coste-beneficio óptima para las empresas al invertir en estas materias y evitar costes de absentismo o presentismo, entre otros.

Asimismo, otro estudio, titulado "Cálculo del rendimiento internacional de la prevención para las empresas: costos y beneficios de las inversiones en seguridad y salud en el trabajo", demuestra que por cada euro invertido por empleado cada año en la prevención en el lugar de trabajo, las empresas pueden esperar un rendimiento económico potencial de 2,20 euros.

Finalmente, se menciona un tercer estudio llevado a cabo por el Instituto de Investigaciones en Salud de PricewaterhouseCoopers (PwC), junto con el Foro Económico Mundial, sobre el programa para la buena condición de salud entre corporaciones multinacionales (más de tres millones de empleados a nivel mundial), en el que se ha demostrado un retorno de la inversión en una relación de 3:1. Las dos principales razones citadas para promover la buena salud fueron «reducir los costes indirectos relacionados con el absentismo, el presentismo y la incapacidad laboral de los trabajadores» $y$ «mejorar el rendimiento laboral, tales como la productividad y calidad». «Reducir los costes directos de la atención sanitaria» fue citada como la tercera razón más importante, seguida en orden de importancia por «el deseo de mejorar la imagen de la empresa internamente (para fidelizar a empleados)», y para «mejorar la imagen de la empresa externamente (captar nuevos talentos)».

Como se puede observar, estos estudios constatan una ratio de retorno de la inversión que oscila sobre los 2,5 a 3 euros por cada euro invertido, como término medio. «Negocio» este en el que podríamos anticipar que cualquier gestor invertiría sin dudarlo.

En otro orden de cosas y dentro de un ámbito más específico de actuación, aunque sin salirse de las actuaciones preventivas, existe un estudio sobre programas preventivos en consumo de drogas en el medio laboral elaborado por el Consorcio Internacional sobre el Abuso de Drogas en el Ámbito Laboral, que demuestra que por cada 0,6 euros invertidos, las empresas pueden esperar un retorno de entre 3 y 9 euros.

Además, se dispone de otro estudio que demuestra una relación de tipo proporcional entre la accidentalidad grave que se acumula en un país y su competitividad. El gráfico siguiente es el resultado de dicho estudio (ver figura 1).

\section{Figura 1. Relación entre competitividad y seguridad por países Competitividad y seguridad}

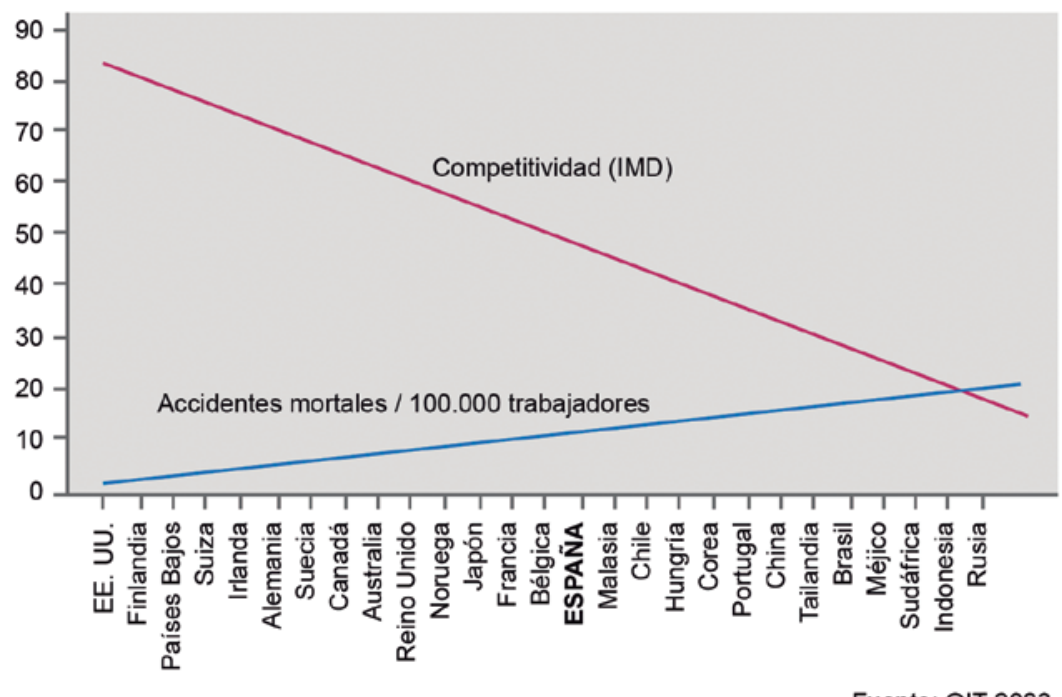

Fuente: OIT 2003 
La relación existente confirma que a mayor número de accidentes graves acaecidos en un país, menor competitividad de dicho país. Estos datos son fiables puesto que los accidentes mortales son evidentes e imposibles de enmascarar u ocultar, además de que la institución que avala el estudio se fundamenta en el International Institute of Management Development de Lausanne (OIT, 2003).

Un estudio más, este llevado a cabo por el Instituto Nacional de Seguridad e Higiene de España, en el año 2011 en cincuenta empresas que se desenvuelven en el camino de la excelencia, sobre la contribución de la eficacia del sistema de prevención de riesgos laborales al éxito empresarial, revela que existe una relación positiva entre empresas que alcanzan resultados significativamente buenos, diferenciándose en las mejores prácticas identificadas en excelencia según el Modelo EFQM (modelo que a través de sus herramientas indica el nivel de excelencia empresarial obtenido en la empresa motivo de estudio a través de diversas dimensiones y una serie de ítems que integran aspectos de calidad, medio ambiente, condiciones de trabajo o responsabilidad social corporativa, entre otros), y las que obtienen también resultados elevados en la gestión preventiva que llevan a cabo sistemáticamente. Las organizaciones que destacan en la excelencia empresarial muestran un nivel más avanzado en la gestión de la prevención y la seguridad y salud de los trabajadores. Se ha observado que existe una correlación positiva entre ambos conceptos, por lo que se puede afirmar que la prevención de riesgos laborales es una condición necesaria irrenunciable para alcanzar la excelencia empresarial (Bestratén y otros, 2013).

El objetivo del estudio realizado en el año 2013 ha sido doble; por un lado, analizar la correlación entre el nivel de calidad del sistema de gestión preventivo y el nivel de gestión de la RSE, demostrado a partir de criterios inspirados en los estándares más relevantes sobre esta materia. Y, por otro, identificar buenas prácticas en empresas que se supone que van más allá de estándares convencionales y de lo legalmente exigible. Independientemente de los resultados obtenidos, la propia metodología aplicada con los cuestionarios diseñados para la identificación y evaluación de responsabilidades puede ser de gran ayuda a cualquier organización que quiera adentrarse en esta materia (ver figura 2).

Figura 2. Relación entre la excelencia empresarial y la prevención de riesgos laborales

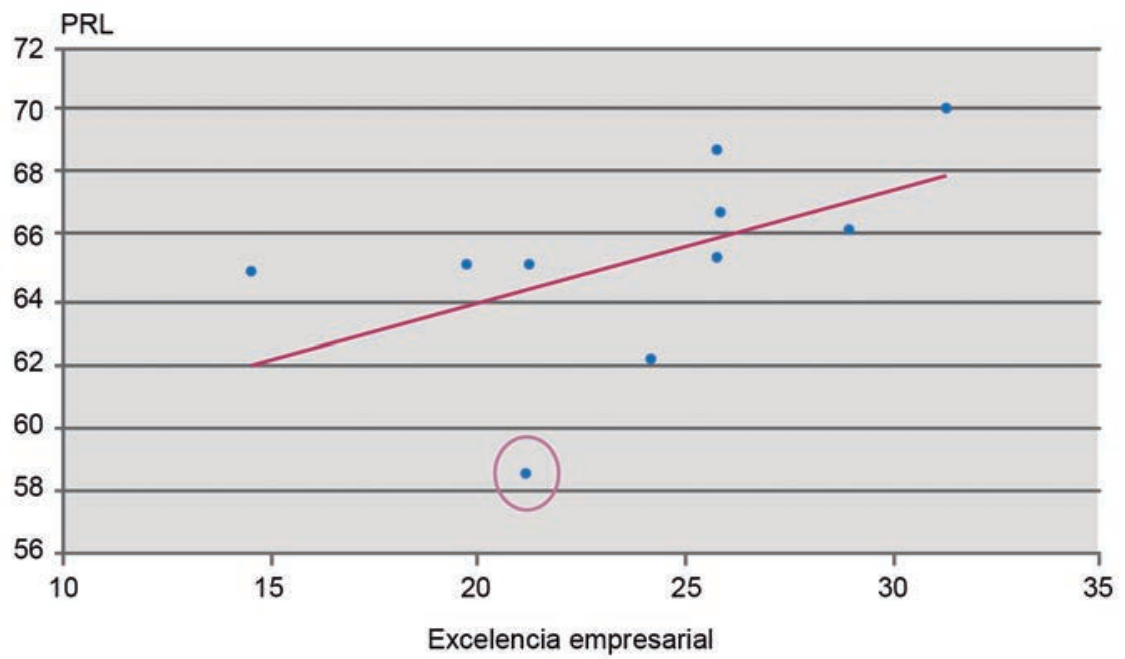

Como colofón final a todos estos estudios mencionados, cabe señalar que los datos internacionales demuestran que entre un 3 y un 10\% del volumen de facturación de una empresa se pierde por las consecuencias económicas de un entorno de trabajo inseguro o no saludable. Ello, una vez más, hace hincapié en la rentabilidad incuestionable de la acción preventiva en las empresas. 


\section{La mejora continua}

En el ámbito de la gestión preventiva en general ha predominado el enfoque humanista de sus resultados. Así, son los indicadores de resultados más utilizados y conocidos aquellos que están vinculados a este enfoque (índices de siniestralidad o de absentismo, por ejemplo); y, por el contrario, resulta difícil disponer de indicadores de resultados socioeconómicos que pudieran habilitar el uso y manejo de estos indicadores (Bestratén y Salas, 2013).

Los sistemas de gestión de las empresas se basan, en cualquiera de sus facetas, en el llamado círculo de mejora continua o círculo de Deming. Este círculo avala el buen funcionamiento de las distintas áreas de gestión (producción, calidad, medio ambiente, salud laboral o seguridad vial, entre otras) y su contenido dispone cuatro fases -planificar, hacer, verificar y actuar (PDCA)-, cuyo desarrollo es fundamental para el éxito de su funcionamiento.

En prevención de riesgos laborales, este círculo, además de ser una guía para su funcionamiento, queda plasmado en las exigencias legales que le afectan. La normativa señala la necesidad de evaluar los riesgos y planificar actuaciones relacionadas con dicha evaluación con el fin de eliminar o controlar los riesgos. Además, la propia ley recoge la necesidad de especificar qué actuaciones va a llevar a cabo la empresa y, asimismo, disponer de una cronología, responsables de ejecución y dotación de recursos para llevarlas a cabo.

De esta forma, existe la obligación de disponer un presupuesto económico y de su distribución específica y, sin embargo, a pesar de que el círculo de Deming implica que deben verificarse las actuaciones para actuar y planificar de nuevo mejorando la gestión a cada giro del propio círculo, en la gestión preventiva no se lleva a cabo tal verificación de los aspectos económicos.

El ACB resulta fundamental en las empresas para poder comprobar si son competitivas y si su productividad responde a las diferentes actuaciones que se van planificando y concretando desde las distintas áreas de gestión.

Si en seguridad y salud laboral no se procede a determinar estas cuestiones, será muy complicado que los gerentes o directivos de las empresas tengan en consideración este ámbito tan importante y estratégico de la gestión empresarial al mismo nivel que otros ámbitos de gestión, tales como calidad o producción, por ejemplo.

\section{Cálculo de costes de los accidentes}

Los accidentes laborales son un tipo de costes que varían según la productividad que tenga la empresa; así, si hay un aumento en la producción, la probabilidad de que se produzcan accidentes podría ser mayor. A estos accidentes se les asignan unos costes directos (que son los costes imputables a su unidad de producción, por ejemplo el salario del personal) y unos costes indirectos (amortización de equipos o maquinarias, por ejemplo), que no son fácilmente imputables a la producción y que se deben igualmente computar (Guixà y otros, 2013).

La tendencia actual es la de simplificar al máximo los análisis de costes de accidentes-incidentes para, una vez tipificados en función del tipo de accidente, características de la empresa, tamaño, nivel tecnológico, competencias de los trabajadores en las diferentes áreas de trabajo, etc., poder hacer estimaciones sencillas y rápidas. Evidentemente, para poder hacerlo aceptablemente habría que haber generado primero experiencia propia en el conocimiento de las causas de los accidentes que suelen acontecer y sus costes normalmente esperados. Otra cosa sucede con los accidentes graves o muy graves, cuyas consecuencias pueden ser cuantiosas y requieren un análisis puntual y minucioso de sus costes. De ahí la importancia de hacer, en las evaluaciones de riesgos, estimaciones de los costes de los daños máximos esperables y concebibles tras un accidente grave.

El cálculo de costes de accidentes puede establecerse con diferentes metodologías, pero la más aplicada es la llamada de «elementos de producción», a través de la cual se recogen los diferentes conceptos que participan en el coste de un accidente, confeccionándose una plantilla tipo que recoge estos conceptos agrupándolos y que, asimismo, puede adaptarse a las necesidades de cada sector y/o empresa que la implemente, para luego poder obtener un importe final que señale la totalidad del coste. Esta plantilla será la base inicial para proceder a integrar el ACB en la gestión habitual de la seguridad y salud en las empresas. 
No se trata de inventar nada, sino de aplicar los conocimientos existentes al campo de la prevención y tener en cuenta las peculiaridades que como área de gestión estratégica se deben plasmar también en la integración del cálculo de costes de la accidentalidad. Estas peculiaridades obligan a utilizar indicadores de gestión socioeconómicos que ayuden a poner en evidencia los costes y demás aspectos preventivos que, de un modo gráfico, se encuentran bajo el agua configurando la masa más importante de todo el iceberg con el que nos encontramos.

\section{Conclusiones}

Los prevencionistas no podemos seguir albergando nuestras mayores dosis de argumentación rentabilista en cuestiones estratégicas o intangibles de forma prioritaria; hemos de buscar el consenso en cuanto a misión y visión entre empresarios y prevencionistas.

Es necesario integrar el cálculo de costes en la gestión habitual en cualquier sistema de gestión de prevención de riesgos laborales, y comenzar con el cálculo de costes de los accidentes puede ser un buen comienzo para hacerlo.

El sistema de costes aplicado debe cumplir una serie de requisitos básicos: ser simple, realista, consensuado, integrado, basado en métodos y conceptos contrastados y adaptable a cada realidad empresarial.

El cálculo de costes permite, a su vez, calcular la rentabilidad desde la productividad, poniendo en evidencia la conveniencia o no de implementar medidas correctoras o preventivas en función de los costes acumulados por una tipología concreta de accidente o de daño a la salud, e incluso demostrándose que el aumento de productividad y la disminución del riesgo (y por lo tanto la disminución de la probabilidad de que se produzcan daños a la salud de los trabajadores expuestos) son compatibles y posibles entre sí.

\section{Bibliografía}

ARCAND, J. L. (2001). undernourishment and economic growth: The efficiency cost of Hunger. Roma: FAO Economic and Social Development WP.

ASOCIACIÓN INTERNACIONAL DE LA SEGURIDAD SOCIAL (2011). "Cálculo del rendimiento internacional de la prevención para las empresas: costes y beneficios de las inversiones en seguridad y salud en el trabajo». En Congreso Internacional sobre Seguridad y Salud en el Trabajo, Estambul.

BESTRATÉN, M.; POY, X.; RUIZ-ESCRIBANO, M. T. (2013). «Eficacia preventiva y excelencia empresarial (I): buenas prácticas en gestión empresarial». En: Notas Técnicas de Prevención (NTP 966), Instituto Nacional de Seguridad e Higiene en el Trabajo.

BESTRATÉN, M.; POY, X.; RUIZ-ESCRIBANO, M. T. (2013). «Eficacia preventiva y excelencia empresarial (II): buenas prácticas en gestión preventiva». En: Notas Técnicas de Prevención (NTP 967), Instituto Nacional de Seguridad e Higiene en el Trabajo.

BESTRATÉN, M.; SALAS, C. (2013). «Análisis coste beneficio en la acción preventiva (I): bases conceptuales». En: Notas Técnicas de Prevención (NTP 982), Instituto Nacional de Seguridad e Higiene en el Trabajo.

BESTRATÉN, M.; SALAS, C. (2013). «Análisis coste beneficio en la acción preventiva (II): estrategias de medición». En: Notas Técnicas de Prevención (NTP 983), Instituto Nacional de Seguridad e Higiene en el Trabajo.

BESTRATÉN, M.; SALAS, C. (2013). «Análisis coste beneficio en la acción preventiva (III): caso práctico». En: Notas Técnicas de Prevención (NTP 984), Instituto Nacional de Seguridad e Higiene en el Trabajo.

BLOOM, D. E.; CANNING, D.; SEVILLA, J. (2001). Health, human capital and economic growth. Génova: Working Group 8, WHO, CMH.

GUIXÀ, J.; SORIANO, J.; SALAS, C.; OTERO, C.; PANCHO, L. (2013). Prevención de riesgos laborales. Barcelona: Oficina de publicacions acadèmiques digitals de la Universitat Politècnica de Catalunya.

ORGANIZACIÓN INTERNACIONAL DEL TRABAJO (2003). «El trabajo peligroso mata a millones y cuesta billones». Revista Trabajo. Núm. 47.

WORLD ECONOMIC FORUM (2007). Global Competitiveness Report 2005-2006. 


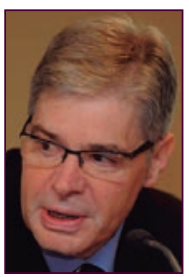

\section{Carles Salas Ollé} carles.salas@upc.edu Transports Urbans i Serveis Generals, SAL

Médico especialista en Medicina del Trabajo por la Universidad de Barcelona y máster en Dirección de los servicios de prevención por la UPC. Técnico superior de Prevención de Riesgos Laborales en las especialidades de Higiene Industrial y de Ergonomía y Psicosociología aplicada por el Instituto Nacional de Seguridad e Higiene en el Trabajo, así como diploma de posgrado en Seguridad e higiene industrial por la Universidad Politécnica de Cataluña, y, finalmente, doctor en Psicología por la Universidad de Valencia. Ha participado y participa habitualmente como ponente en foros nacionales e internacionales en prevención de riesgos laborales. Ha publicado libros y artículos sobre salud laboral y prevención de riesgos laborales en diversas revistas especializadas en la materia. Cuenta con una experiencia en este campo de cerca de veinte años. Desarrolla labores como asesor, docente y auditor y, en particular, como profesor asociado e investigador del Departamento de Organización de Empresas de la Universidad Politécnica de Cataluña (Cerpie). Ha participado en la promoción e impulso de la seguridad y salud laboral y las condiciones de trabajo, a través de intervenciones en programas de diversos medios de comunicación audiovisual de primer orden.

Los textos publicados en esta revista están -si no se indica lo contrario- bajo una licencia Reconocimiento-Sin obras derivadas 3.0 España de Creative Commons. Puede copiarlos, distribuirlos y comunicarlos públicamente siempre que cite su autor y la revista y la institución que los publica (autoría, nombre de la revista, institución editora); no haga con ellos obras derivadas. La licencia completa se puede consultar en http://creativecommons.org/licenses/by-nd/3.0/es/deed.es.

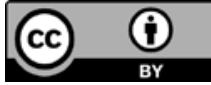

